\title{
Yucca gloriosa
}

\section{(Spanish Dagger, Mound-Lily Yucca)}

Just like Y. aolifolia this yucca is an erected evergreen shrub with sword-like leaves originating from a basal rosette . Leaf margins on Spanish Dagger (ucca gloriosa)Y are smooth, whereas those on Yucca aloifolia (Spanish Bayonet) are rough. The outer half of the leaves on Spanish Dagger also bends toward the ground, whereas those on Spanish Bayonet do not. Also the leaf color for the mound-lily is more grayish- greenish whereas for the Spanish Bayonet its always dark green. During summer time its produces its white-purplish fragrant spike of flowers. Mound-lily yucca does best in full sun with sandy, very well drained soil, either neutral or acidic. Very drought tolerant. Excellent for xeriscaping. Used as a specimen or as a foundational plant. As it does not have a needle- sharp leaf tip like the Spanish bayonet uit can be used more commonly in garden designs.

\section{Landscape Information}

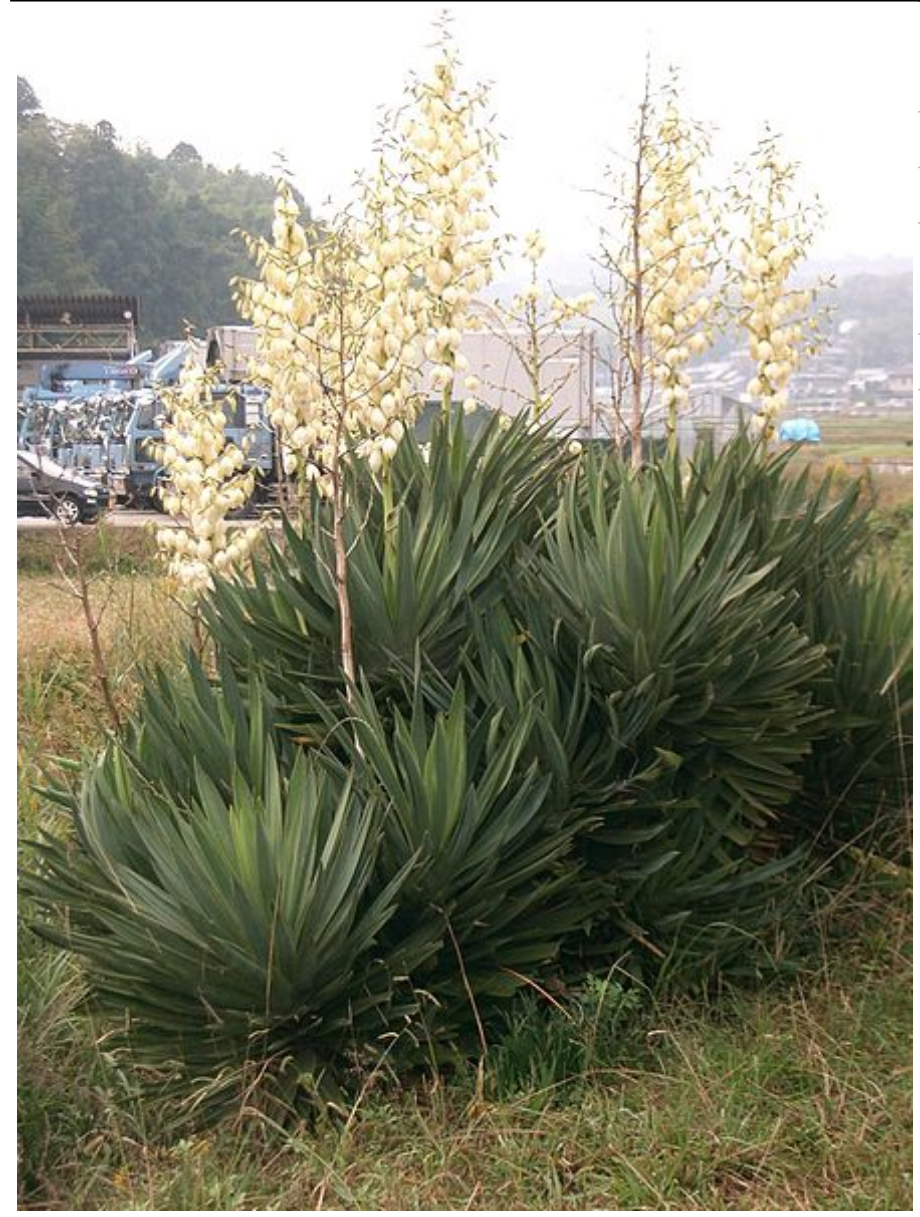

French Name: Yucca glorieux, Yucca acuminé Pronounciation: YUCK-kuh glor-ree-OH-suh Plant Type: Shrub Origin: Florida Heat Zones: 7, 8, 9, 10, 11, 12 Hardiness Zones: 7, 8, 9, 10, 11

Uses: Screen, Hedge, Specimen, Wildlife Size/Shape

Growth Rate: Slow

Tree Shape: Round

Canopy Symmetry: Symmetrical

Canopy Density: Medium

Canopy Texture: Coarse

Height at Maturity: Less than $0.5 \mathrm{~m}$

Spread at Maturity: 1.5 to 3 meters

Time to Ultimate Height: 2 to 5 Years 
Botanical Description

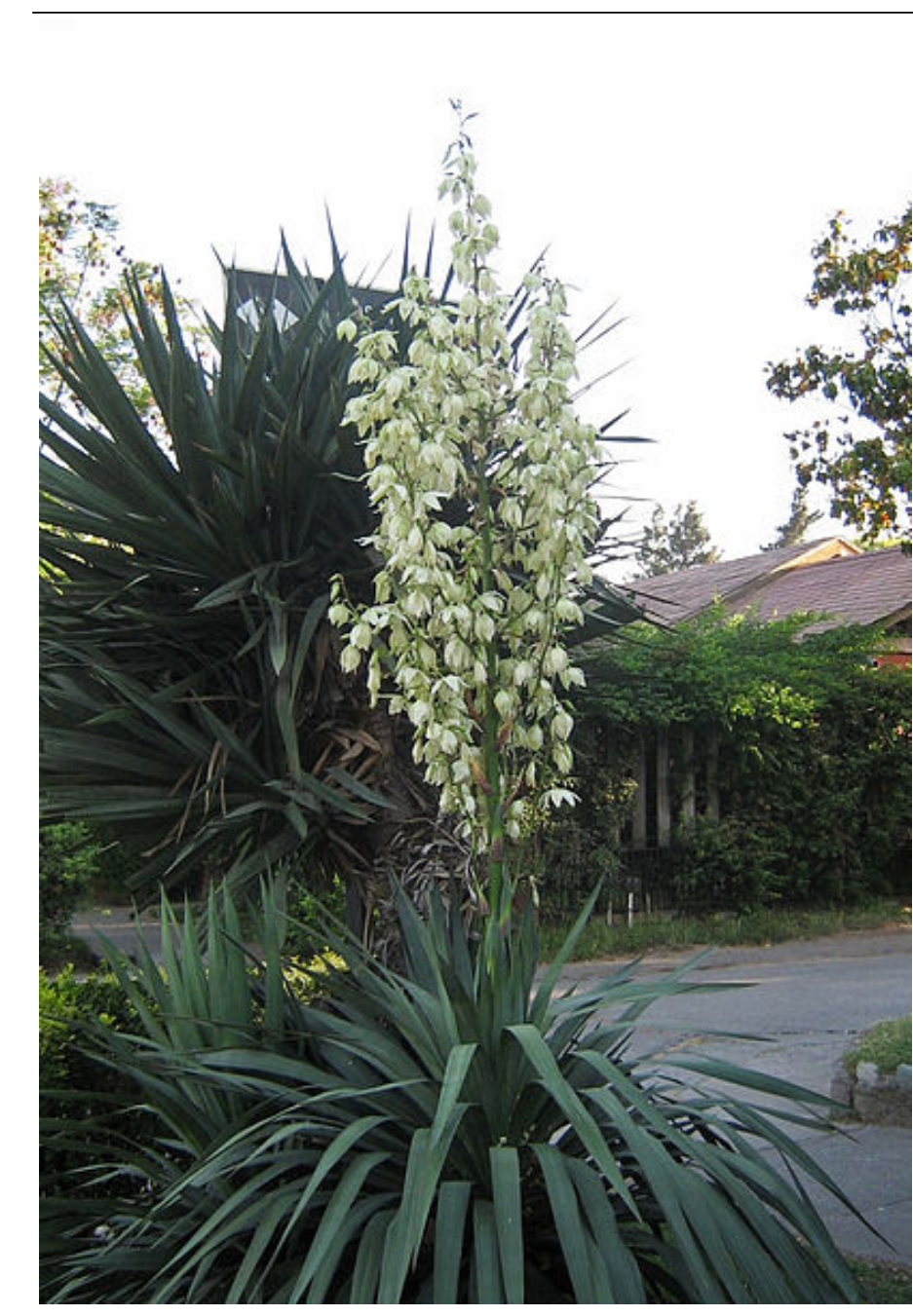

Flower Image

\section{Foliage}

Leaf Arrangement: Alternate

Leaf Venation: Parallel

Leaf Persistance: Evergreen

Leaf Type: Simple

Leaf Blade: 30 - 50

Leaf Shape: Linear

Leaf Margins: Entire

Leaf Textures: Rough

Leaf Scent: No Fragance

Color(growing season): Green, Blue-Green

Color(changing season): Green

Flower

Flower Showiness: True

Flower Size Range: Over 20

Flower Type: Spike

Flower Sexuality: Monoecious (Bisexual)

Flower Scent: Pleasant

Flower Color: White

Seasons: Summer

Trunk

Trunk Susceptibility to Breakage: Generally resists breakage

Number of Trunks: Can be trained to one trunk Fruit

Fruit Type: Capsule

Fruit Showiness: True

Fruit Size Range: 0 - 1.5

Fruit Colors: Black

Seasons: Summer 


\section{Yucca gloriosa}

(Spanish Dagger, Mound-Lily Yucca)

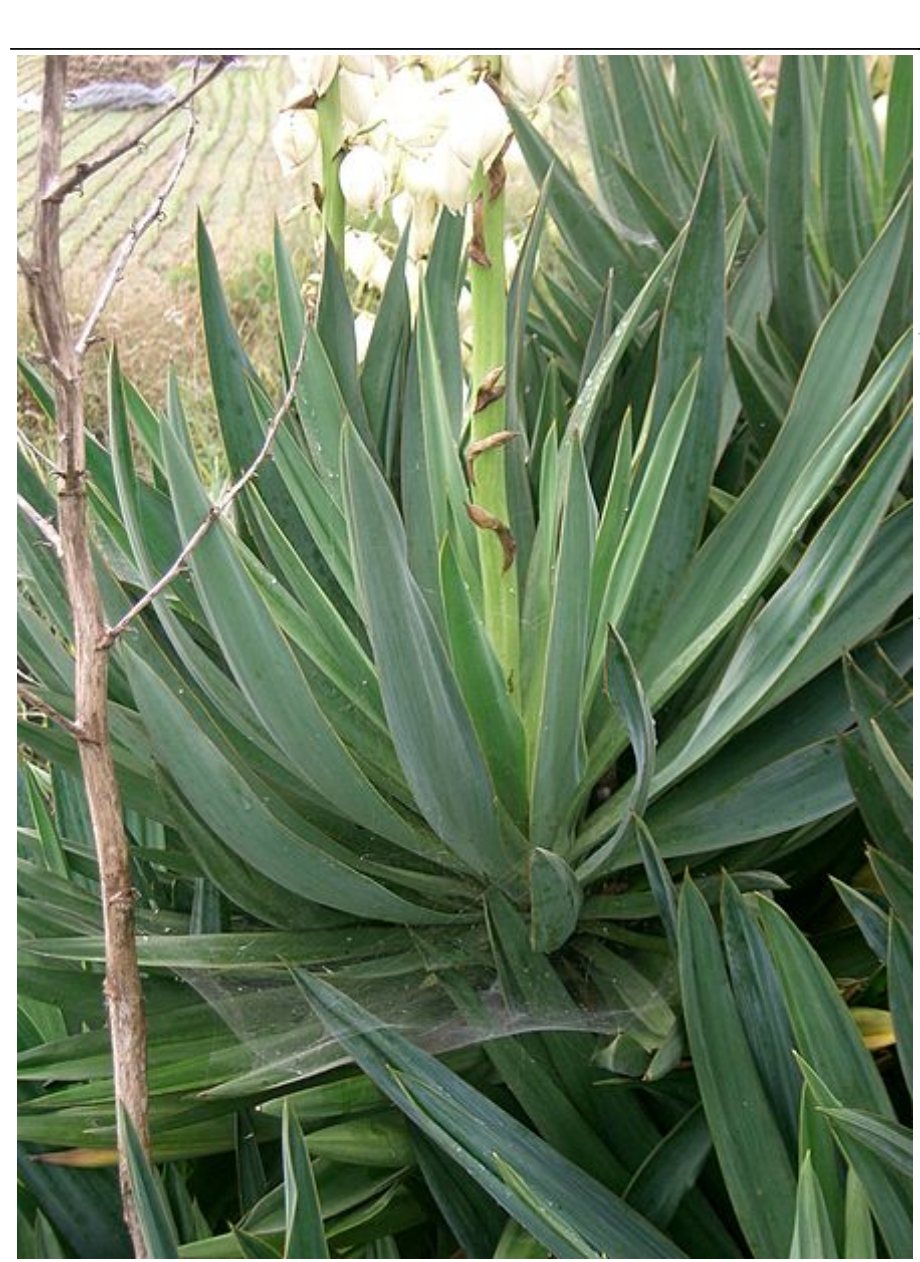

Horticulture Management

Tolerance

Frost Tolerant: Yes

Heat Tolerant: Yes

Drought Tolerant: Yes

Salt Tolerance: Moderate

Requilrements

Soil Requirements: Sand

Soil Ph Requirements: Neutral

Water Requirements: Moderate, Low

Light Requirements: Full, Part

Management

Susceptibility to Pests and Diseases: No

Pruning Requirement: No pruning at all

Fruit/ Leaves/ Flowers litter: No

Surface Rooting: Yes

Life Span: 25-50 years

Edible Parts:

Pests: Scales

Plant Propagations: Cutting, Layering 


\section{MORE IMAGES}

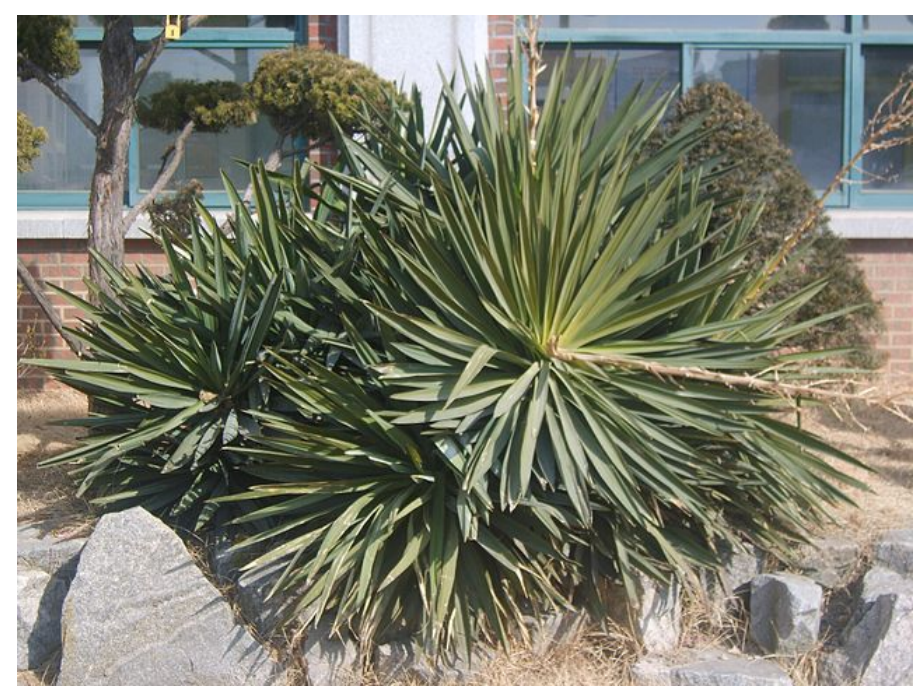

Other Image 